\title{
Few-layer graphene integrated tilted fiber grating for all-optical switching
}

\author{
Biqiang Jiang, Yueguo Hou, Hongyang Wang, Xuetao Gan, Ailun Li, Zhen Hao, Kaiming Zhou, Lin \\ Zhang, and Jianlin Zhao
}

\begin{abstract}
Recently, the integration of two-dimensional materials with optical fibers has opened up a great opportunity to develop all-fiber signal-processing devices. Graphene is an ideal material for all-optical signal processing via thermal-optic effect because of its high electrical and thermal conductivity, as well as broadband light-matter interactions with fast responses. Herein, we report the achievement of all-optical switching with fast response by integrating few-layer graphene onto a tilted fiber Bragg grating (TFBG) inscribed in a reduceddiameter fiber. Relying on graphene's decent photothermal effect, the transmission spectrum of the TFBG could be alloptically modulated by tuning the incident pump power. The all-optical switch can consequently operate at a series of wavelengths owing to the TFBG's comb-like resonances. The reduced diameter of the graphene-integrated TFBG and the pump at its resonant wavelength promise the alloptical switch to have a fast-dynamic response of around 1 $\mu$ s and an extinction ratio exceeding $13 \mathrm{~dB}$. This compact device with graphene integration has the potentials to be integrated into all-fiber system to extend the functions of all-optical signal processing.
\end{abstract}

Index Terms-All-optical switch, few-layer graphene, reduceddiameter fiber, tilted fiber grating.

\section{INTRODUCTION}

$\mathrm{A}$ 1l-optical switches in fibers are intriguing for optical interconnect and signal processing [1, 2]. All-optical schemes can overcome the "electrical bottleneck" and avoid electrical-optical-electrical conversions, thereby rendering higher modulation efficiency and lower noisy [3, 4]. Also, an all-in-fiber switch or modulator is more desirable to be compatible with a standard fiber-optic system in real applications. Unfortunately, limited by silica's intrinsically low nonlinear optical responses, significantly high optical powers, large fiber lengths, or high chalcogenide/ytterbium doping, are required to induce moderately strong all-optical effects in

This work was supported in part by the National Natural Science Foundation of China under Grants 61775182, 61975166, 61775183, and in part by the Natural Science Basic Research Plan in Shaanxi Province of China under Grant 2019JM330. (Corresponding authors: Xuetao Gan; Jianlin Zhao).

B. Jiang Y. Hou, H. Wang, X. Gan, A. Li, Z. Hao, and J. Zhao are with the MOE Key Laboratory of Material Physics and Chemistry Under Extraordinary Conditions and Shaanxi Key Laboratory of Optical Information Technology, optical fibers for all-optical switches [5-7].

Attributed to the leaking evanescent field of geometrymodified fibers, including microfibers [8-12], side-polished fibers [13, 14], and etched/tilted fiber Bragg gratings (FBGs) $[15,16]$, it is possible to integrate active materials with them to assist the all-optical effects. For instance, by using the optical Kerr effect [8] and absorption loss effect [2, 9], the graphenecoated microfiber can be used to achieve the ultrafast all-optical modulation, while showing a low modulation depth and requiring the pump lasers with high-peak power. With the strong interactions between evanescent field of microfibers and the graphene, tungsten disulfide $\left(\mathrm{WS}_{2}\right)$, as well as Au nanorods, high-efficiency thermal-optic devices are developed for alloptical switches [10-12]. In these coated fiber devices, the photothermal effect was employed to heat the fiber and give rise to phase shift of the guiding modes in fibers [10]. To realize the switch of optical intensities, Mach-Zehnder interferometers (MZIs) or Michelson interferometer are required to convert the phase change into the amplitude variation. Also, to reduce the pump power and loss, the external pumping through free space is employed to heat one arm of the graphene-coated fiber MZI for all-optical switches [17]. However, off-axis interferometers would be susceptible to disturbance, such as the ambient weak vibrations, hindering them from practical applications. Etched FBG coated with graphene could realize the optical switch directly due to the distributed feedback [15], while the optical signal could be switched only at a specific Bragg wavelength. It is difficult to work at a broad wavelength range. Meanwhile, in the above devices, they have the response time in the order of millisecond or even seconds caused by the photothermal effect, and the enhancement of the light-matter interaction is at the expense of the loss induced by the coating photothermal layer as well as the mechanical strength of fibers.

In this reported work, we propose an all-optical switch by integrating few-layer graphene onto a tilted FBG (TFBG) inscribed in a thin fiber with the reduced diameter. Unlike interferometer-based optical switches, the TFBG has a compact and stable structure, and the slanted grating planes in the thin fiber will excite a spectral comb of narrowband-cladding mode

School of Physical Science and Technology, Northwestern Polytechnical University, Xi'an 710129, China (email: bqjiang@nwpu.edu.cn; yueguohou@mail.nwpu.edu.cn; whyyyyt@163.com; xuetaogan@nwpu.edu.cn; alanlitell@163.com; zhenhao@mail.nwpu.edu.cn; jlzhao@nwpu.edu.cn).

K. Zhou and L. Zhang are with the Aston Institute of Photonic Technologies, Aston University, Birmingham B4 7ET, UK. (email: k.zhou@aston.ac.uk; 1.zhang@aston.ac.uk). 
resonances in a wide wavelength range [18-21]. Hence, the thin TFBG allows the all-optical operation in a series of wavelengths where light has been coupled out of the core. Graphene has the extremely high electrical and thermal conductivity of the graphene, and then promises a very fast switching rate for all-optical switch or modulator via thermaloptic effect [22, 23]. The spectrum of the TFBG could therefore be all-optically modulated. In a similar scheme reported recently [16], there are a slow modulation rate with watt-level pump power by observing the self-starting pulsing response, and low pump-heating efficiency due to the non-resonant pump with a broadband light. By contrast, the resonant-pump at a dip wavelength of the TFBG's cladding mode and the employment of the thin fiber, will significantly enhance the accessible evanescent field and the pump efficiency, and promote a strong pump-heating-caused thermal interaction with graphene. Moreover, compared with the schemes using geometrymodified fibers embedded in interferometers, the grapheneintegrated TFBG presents a fast response of around $1 \mu \mathrm{s}$, an extinction ratio exceeding $13 \mathrm{~dB}$ and a low pump power of less than $45 \mathrm{~mW}$ required. Its compact and robust structure makes it easier to be incorporated into an all-fiber signal processing.

\section{Device Preparation And Characterizations}

The employed TFBG has the slanted grating planes, which enhances the light coupling from the forward-propagating core mode to a series of backward-propagating cladding modes, and simultaneously reduces the coupling to the backwardpropagating core mode. According to the phase-matching conditions, the resonant wavelengths of the core mode $\left(\lambda_{\mathrm{co}}\right)$ and cladding modes $\left(\lambda_{\mathrm{cl}}\right)$ of the TFBG can be expressed as

$$
\begin{gathered}
\lambda_{\mathrm{co}}=2 n_{\mathrm{eff}}^{\mathrm{co}}\left(\lambda_{\mathrm{co}}\right) \Lambda / \cos \theta, \\
\lambda_{\mathrm{cl}}=\left[n_{\mathrm{eff}}^{\mathrm{co}}\left(\lambda_{\mathrm{cl}}\right)+n_{\mathrm{eff}}^{\mathrm{cl}}\left(\lambda_{\mathrm{cl}}\right)\right] \Lambda / \cos \theta,
\end{gathered}
$$

where, $n_{\text {eff }}^{\text {co }}\left(\lambda_{\mathrm{co}}\right)$ and $n_{\text {eff }}^{\text {co }}\left(\lambda_{\mathrm{cl}}\right)$ are the effective refractive indices of the core mode at $\lambda_{\mathrm{co}}$ and $\lambda_{\mathrm{cl}}$, respectively, $n_{\mathrm{eff}}^{\mathrm{cl}}\left(\lambda_{\mathrm{cl}}\right)$ is the effective index of the cladding mode at $\lambda_{\mathrm{cl}}, \theta$ is the tilt angle, $\Lambda$ is the nominal grating period measured perpendicular to grating planes. Also, from Eq. (2), the integration of layered materials will modulate the effective refractive index and the resonant wavelengths of the TFBG's cladding modes.

Figure 1(a) schematically depicts the graphene-integrated TFBG and its operation principle for all-optical switching. The employed TFBG was UV-inscribed in a thin fiber by using a frequency doubled continuous wave $\mathrm{Ar}^{+}$laser $(244 \mathrm{~nm})$ and the scanning phase-mask technique. The hydrogenated thin fiber has the reduced core/cladding diameter of $4.2 / 80 \mu \mathrm{m}$ for the enhancement of the photosensitivity and evanescent field. The length, tilted angle, and period of the inscribed grating are 15 $\mathrm{mm}, 9.0^{\circ}$, and $\sim 536 \mathrm{~nm}$, respectively. The employed graphene film was grown on a copper foil by chemical vapor deposition and had a five-layer thickness on the foil to provide stronger interaction with the cladding modes of thin TFBG than the monolayer graphene. The layer number was distinguished by a Raman spectroscope and transmission electron microscope [24].
Then the copper foil was chemically etched, leaving a five-layer and $\sim 15 \mathrm{~mm}$ long graphene film floating on top of the water. Further, the few-layer graphene film was wrapped around the TFBG by a wetting transfer technique [25].

Figure 1(b) displays the optical microscopic image of the graphene-integrated TFBG under bright-field, showing the uniform graphene coating. By launching a visible red light of $635 \mathrm{~nm}$ into the device, the TFBG with a graphene coating is captured by using the microscope under the dark-field, as shown in Fig. 1(c). The bright red scattered line indicates that the cladding mode is coupled into the graphene layer for the light-matter interaction. From the zoomed white dash-line region of Fig. 1(c), we observe clear diffraction fringes on the TFBG surface shown in Fig. 1(d). The surface of the TFBG with graphene coating was examined by scanning electron microscopy (SEM), as shown in Fig. 1(e). The result shows a relatively uniform graphene coating, but with some slightly wrinkles around the TFBG.
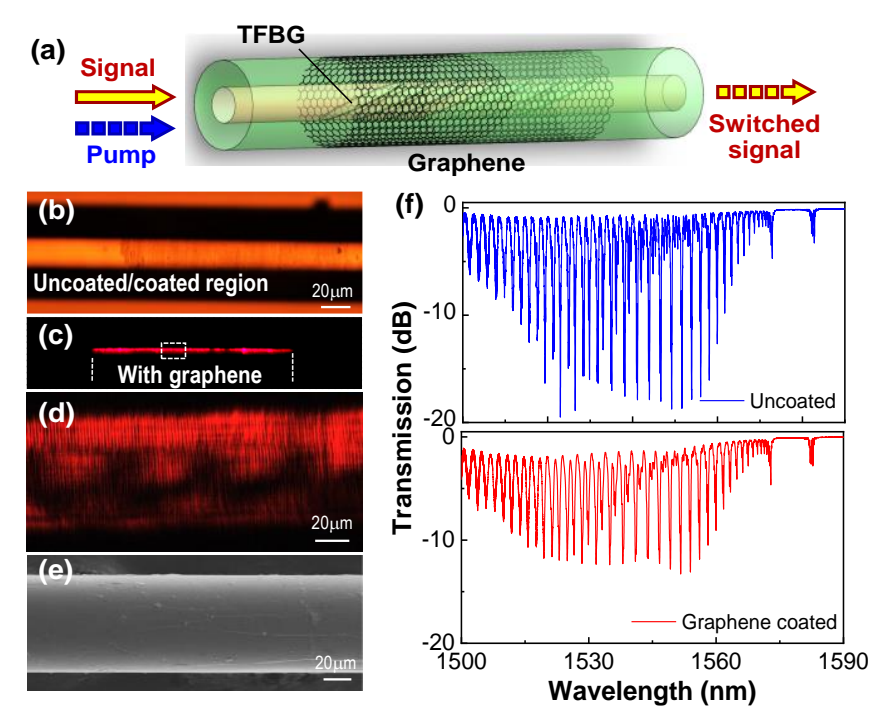

Fig. 1. (a) Schematic of the graphene-integrated TFBG in a reduced-diameter fiber. (b) and (c) Optical microscopic images of the device under bright- and dark-field when launched by a $635 \mathrm{~nm}$ laser. The bright scattered line indicates the graphene-integrated region. (d) Diffraction fringes on the TFBG surface from the area in white dash-line of (c). (e) SEM image of the device. (f) Transmission spectra of the TFBG without and with graphene integration.

The transmission spectra of the TFBG before and after graphene coating are measured using a broadband ASE source and an optical spectrum analyzer (OSA), as depicted in Fig. 1(f). We can observe a set of comb-like cladding mode resonances in a broad wavelength region, covering the whole telecom Cband, as well as the coupling intensity of around $20 \mathrm{~dB}$. In the presence of a few-layer graphene, for the cladding mode around $1549.2 \mathrm{~nm}$, the resonant intensity has an attenuation of $\sim 5.94$ $\mathrm{dB}$, and the resonant wavelength slightly red-shifts by $0.028 \mathrm{~nm}$, as a result of the high complex refractive index of the graphene. The increase of the graphene layer thickness will enhance the light-matter interaction, through at the expense of the coupling intensity of cladding modes and the determined extinction ratio $[10,25,26]$. Therefore, in the experiment, the five-layer graphene was chosen to balance the pump-heating efficiency 
and the extinction ratio of the all-optical device.

\section{RESUlTS AND DISCUSSIONS}

To observe the all-optically controlled spectrum, a narrowband light from a tunable laser (TL) via an erbium-doped optical fiber amplifier (EDFA) was coupled into the grapheneintegrated TFBG as a pump light, accompanying a broadband light from an ASE source with only $0.1 \mathrm{~mW}$ power. By increasing the pump power incident into the device, the transmission spectrum was monitored with the OSA. Figure 2(a) displays the spectral evolution with different powers of the $1554.37 \mathrm{~nm}$ pump light. The spectrum has a global red-shift as the power increases, and at a given wavelength, the contrast of the cladding mode resonances is up to $13 \mathrm{~dB}$, which is possible to implement a high-performance optical switch. As an example, the gradually red-shifted resonant transmission dip around the wavelength $1550 \mathrm{~nm}$ is shown in the inset of Fig. 2(a). In detail, the initial wavelengths (and $3 \mathrm{~dB}$ bandwidths) of three resonant dips (Dip 1, 2, and 3) marked with star symbols are 1526.713 $\mathrm{nm}(0.27 \mathrm{~nm}), 1546.885 \mathrm{~nm}(0.16 \mathrm{~nm})$ and $1558.306 \mathrm{~nm}(0.10$ $\mathrm{nm})$, respectively. As the incident pump power increases, the wavelengths of the dips nonlinearly red-shift by $\sim 0.8 \mathrm{~nm}$ with the incident pump power, and then approach to be stable, as shown in Fig. 2(b). In the power range of 0-45 mW, the resonant wavelength linearly red-shifts by $\sim 0.3 \mathrm{~nm}$ redshift with a response coefficient of $6.7 \mathrm{pm} / \mathrm{mW}$, changing the dip to the plateau in the spectrum of cladding mode resonance, and the attenuation of the cladding mode resonance attenuation by $\sim 2.4$ $\mathrm{dB}$ with a response coefficient of $0.054 \mathrm{~dB} / \mathrm{mW}$.

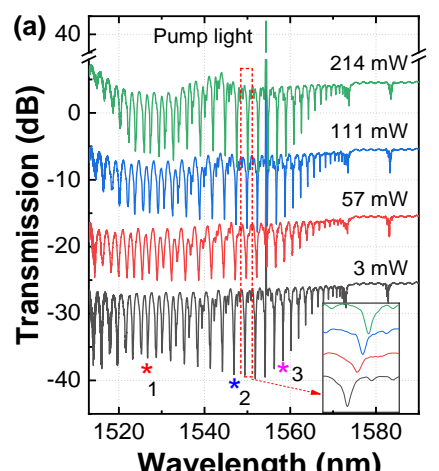

Wavelength (nm)

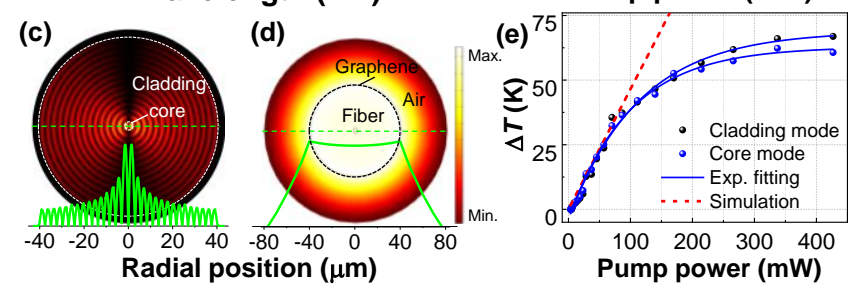

Fig. 2. (a) Spectral evolution of the graphene-integrated TFBG with the increased pump power. For clarity, the spectra are shifted vertically. The inset shows the shift of the resonant wavelength near $1550 \mathrm{~nm}$. (b) Resonant wavelengths of different order cladding modes versus the pump power. (c) Modal distribution of the cladding mode $\left(\mathrm{LP}_{1,15}\right)$ of the thin TFBG. (d) steadyheat distributions of the TFBG cross section, where the bottom insets are the corresponding distributions along the radial direction marked with green dashed lines. (e) Temperature changes (black and blue balls) calculated from the wavelength shifts of the cladding mode (Dip 2) and core mode and from the numerical analysis of the pump-heating process.
The above observations can be explained that the graphene produces heat by absorbing the evanescent wave of the pump light to change the local temperature of the TFBG. The pumpheating will modulate the effective refractive index of cladding modes and then induce a globally spectral shift. To explain it, we numerically analyzed the cladding mode resonance pumped thermal process in the graphene-integrated thin TFBG, based on the heat-generation and transfer theory $[10,13,17]$. The temperature distribution $T(r)$ in the thin fiber is described by the steady-state heat conduction equation $-k \nabla^{2} T(r)=Q_{\mathrm{S}}$, where $k=1.38 \mathrm{~W} \cdot \mathrm{m}^{-1} \cdot \mathrm{K}^{-1}$ is the thermal conductivity of silica, and $Q_{\mathrm{s}}$ is the heat source per unit volume generated by graphene absorption. The heat source $Q_{\mathrm{s}}$ can be calculated from the model electric field $\mathbf{E}$ in the graphene layer using $Q_{\mathrm{S}}=1 / 2 \varepsilon_{0} \omega \operatorname{Im}\left(\varepsilon_{\mathrm{r}}\right)|\mathbf{E}|^{2}$, where $\varepsilon_{0}$ is the permittivity of vacuum, $\omega$ is the angular frequency of light, and $\mathcal{E}_{\mathrm{r}}$ is the relative permittivity of graphene. Due to the thin graphene layer and high thermal conductivity $\left(5300 \mathrm{~W} \cdot \mathrm{m}^{-1} \cdot \mathrm{K}^{-1}\right)$, the graphene coating can be considered as a uniform surface heat source. By using the simulated electric field distribution of the 15th cladding mode ( $\left.\mathrm{LP}_{1,15}\right)$ shown in Fig. 2(c), corresponding to the pump wavelength in the experiment, we calculated the steadystate temperature distribution in the thin TFBG by using the finite element method. The results are shown in Fig. 2(d), taking into account the axial/radial heat transfer in the fiber and air. The fiber exhibits a small temperature gradient distribution along the radial direction. Also, the temperature gradient will increase with increasing of the pump-heating in the fiber boundary.

Hence, we further solved the temperature change in the fiber as a function of the pump power to estimate the experimental results. When the heat source determined by $|\mathbf{E}|^{2}$ is calibrated according to the modal distribution at initial pump wavelength, the numerically simulated result is plotted with the red dashed line in Fig. 2(e). The temperature linearly rises with the increase of the pump power. Meanwhile, we also measured the temperature response of the TFBG's spectrum by placing the device in a temperature controllable chamber. Hence, the temperature changes at different pump powers can be evaluated according to the measured wavelength shifts of the cladding mode (Dip 2) and core mode, as well as their temperature response coefficients $\left(\mathrm{d} \lambda / \mathrm{d} T=11.81 \mathrm{pm} \cdot \mathrm{K}^{-1}\right.$ for Dip 2 and 12.36 $\mathrm{pm} \cdot \mathrm{K}^{-1}$ for core mode) and the corresponding thermal-optic coefficient $\left(\mathrm{d} n / \mathrm{d} T=\sim 10^{-5} \mathrm{~K}^{-1}\right)$. The results show that the dependences of effective refractive indices of the cladding mode and core mode with pump power are $5.7 \times 10^{-6} \mathrm{~mW}^{-1}$ and $5.4 \times 10^{-6} \mathrm{~mW}^{-1}$, respectively. In the low-power region below 80 $\mathrm{mW}$, the results marked with black and blue balls in Fig. 2(e) agree well with the simulated results. However, as the pump power gradually increased over $80 \mathrm{~mW}$, the pump wavelength is far away from that of the cladding mode resonance, and then, the intensity of the evanescent field in the graphene layer will decrease, resulting in a reduced pump-heating. Therefore, there is a growing difference between the simulated and measured results in the high-power region. Eventually, the generation and dissipation of Joule heat will reach equilibrium, and the 
temperature will almost not increase when the pump power is more than $250 \mathrm{~mW}$. Moreover, from Fig. 2(e), there is a small difference between the temperatures calculated from the cladding and core modes in the high pump-power region, which is attributed to the non-ignorable temperature gradient inside the fiber. Therefore, the accessible evanescent field in the thin fiber and the pump-heating efficiency will be greatly enhanced due to the light circulating when the pump wavelength matches with that of the cladding mode resonance at the low pump power. As a result, in comparison with the non-resonant pump in the previous reports $[16,27]$, the pump power is reduced by 1-2 orders of magnitude to achieve the same spectrum shift, which can also help to reduce the response time of the alloptically controlled spectrum [28]. It is worth noting that, the pump-heating the pump-heating efficiency could be further improved by using the S-polarized (tangential electric field at the fiber surface) pump laser due to more absorption on the evanescent field [16]. Moreover, in practical applications, when the power of the signal light such as the ASE source is sufficient large, we have to adjust the pump wavelength to match the dip wavelength of the TFBG in advance, since the signal light itself would lead to a considerable spectrum shift.

To examine the all-optical switching effect of the grapheneintegrated TFBG, we established an experimental setup by periodically pumping it and real-time monitoring the transmissions of the pump and signal light, as schematically illustrated in Fig. 3(a). The pump and signal light were combined into the device through a 50:50 fiber coupler. In the measurement, the polarized pump light was supplied by an amplified TL with an EDFA, and modulated by a polarization controller (PC) and an acoustic optical modulator (AOM). The incident power into the device was directly monitored with a powermeter. To evaluate the broadband response of all-optical switching effect, three commercial FBGs as reflection filters were used to acquire the shifts of cladding modes and the changes of coupling intensities at different wavelengths. The reflected spectra of FBGs are shown in Fig. 3(b), and their Bragg wavelengths are $1529.6 \mathrm{~nm}, 1549.8 \mathrm{~nm}$, and $1554.8 \mathrm{~nm}$ (marked with $1530 \mathrm{~nm}, 1550 \mathrm{~nm}$ and $1555 \mathrm{~nm}$ below for simplification), respectively. The filtered signal was monitored by a photodetector (PD) via a fiber circulator. As displayed in Fig. 3(c), when the Bragg wavelength of an FBG is equal to or far from that of the cladding mode resonance, the PD will output a minimum or maximum signal, corresponding to the "off" and "on" states of the signal. To ensure the initial "on" or "off" state, we adjusted the Bragg wavelength of the FBG to align that of the cladding mode resonance by stretching the filtered FBG. The periodical change of the signal was real-time displayed and collected by an oscilloscope. To avoid the disturbance of pump light, the wavelength of the FBG filter is chosen to be far away from that of the pump light.

We measured the transmission spectra of the TFBG by the OSA when the pump was turned off and on with an incident power of $50 \mathrm{~mW}$, as shown in Fig. 3(c). The measured total loss of the pump power through the device is $\sim 9.1 \mathrm{~dB}(\sim 87.7 \%)$. It can be observed that the spectrum has a redshift of $0.35 \mathrm{~nm}$, and the maximum extinction ratio of the coupling intensity is more than $13 \mathrm{~dB}$. The coupling intensity has a slight decrease due to the modulation of the pump light on the imaginary part of the graphene's refractive index [16]. Accordingly, the reflection spectrum of the $\mathrm{FBG}_{2}$ has an obvious attenuation induced by the spectral shift of the TFBG. As a result, with the pump off and on, the output signal from the PD will appear the maximum and minimum values, corresponding to the "on" and "off" states, which provides the feasibility to implement the all-optical switching. From Fig. 3(c), there is a splitting spectrum observed at the $\mathrm{FBG}_{2}$ ' $\mathrm{s}$ "off" state, attributed by the narrower bandwidth of the cladding mode than that of FBG's core mode.

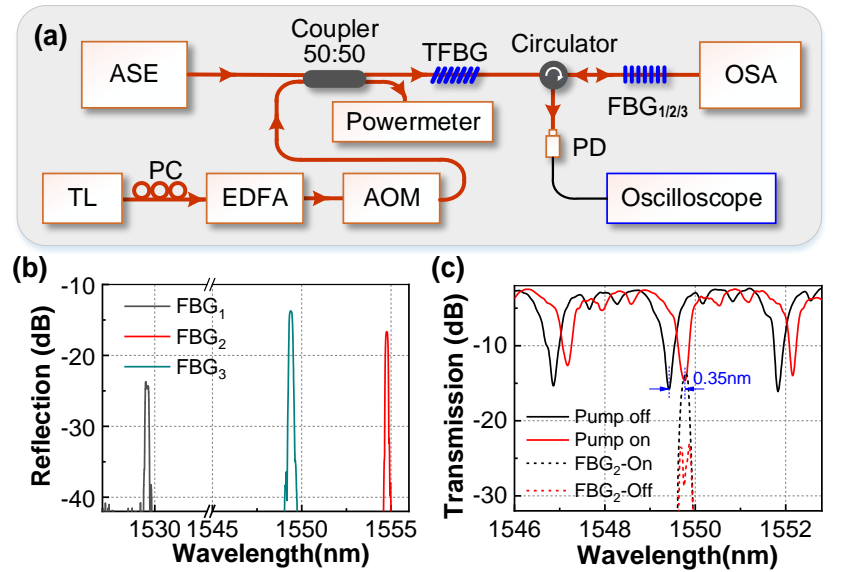

Fig. 3. (a) Schematic of the experimental setup for measuring all-optical switching effect in a graphene-integrated TFBG. (b) Spectra of the employed FBGs for signal filtering. (c) Measured TFBG and $\mathrm{FBG}_{2}$ spectra without (black) and with (red) $50 \mathrm{~mW}$ pump light, corresponding to "on" and "off" states of the signal.

To investigate the dynamic response of the all-optical switching, the pump light was modulated by the AOM to provide a square pump pulse launched further into the graphene-integrated TFBG, and a high-speed InGaAs detector was used to detect the modulated signal intensity. Figure 4(a) shows the detected temporal signals at the modulation frequencies of $1 \mathrm{kHz}, 50 \mathrm{kHz}, 200 \mathrm{kHz}, 500 \mathrm{kHz}$, and $1 \mathrm{MHz}$, where the output intensity of the signal light reflected by the $\mathrm{FBG}_{3}$ at $1550 \mathrm{~nm}$ was monitored by switching the pump on and off. As shown in Fig. 4(a), the waveform of the detected signal is almost the same as that the modulation wave below $200 \mathrm{kHz}$. We also calculated the time taken for the signal to change from $10 \%(90 \%)$ to $90 \%(10 \%)$ of the rising (falling) edge of the 200 $\mathrm{kHz}$ signal wave, which is normally defined in analog signal processing. The results show the rise/fall time of $1.1 / 0.6 \mu \mathrm{s}$, following the $10 \%-90 \%$ rule. By further increasing the modulation frequency of the pump light, the output signal of the device can reach the half of the maximum amplitude at the modulation frequency of $1 \mathrm{MHz}$. Affected by the modulation depth and the sampling of the switched signal, the rise/fall time could be altered at other modulation frequencies or selected wavelength channel, while the response time is always observed in the order of the microsecond.

As depicted in Fig. 1(f), the graphene-integrated TFBG has a set of comb-like transmission spectrum in the whole $\mathrm{C}$ telecom band, and the spectral spacing is determined by the wavelength difference between adjacent mode resonances. The device 
therefore enables us to achieve the all-optical switching at multi-wavelength channels, corresponding to the resonant wavelengths of the TFBG. To verify this, the three FBGs as reflecting bandpass filters were utilized to extract the changes of the coupling intensity of the cladding modes at three different wavelengths, i.e. $1530 \mathrm{~nm}, 1550 \mathrm{~nm}$ and $1555 \mathrm{~nm}$, respectively. For each specific wavelength, the cladding mode resonance of the TFBG would have the simultaneous and similar mode modulation when pumped at a given resonant wavelength. Taking the modulation frequency of $50 \mathrm{kHz}$ as an example, Fig. 4(b) displays the measured temporal responses of the all-optical switching with the same pump power. Clearly, the output signals almost have the same waveform. The device can therefore be implemented in multi-wavelength channels for multiple output processing.
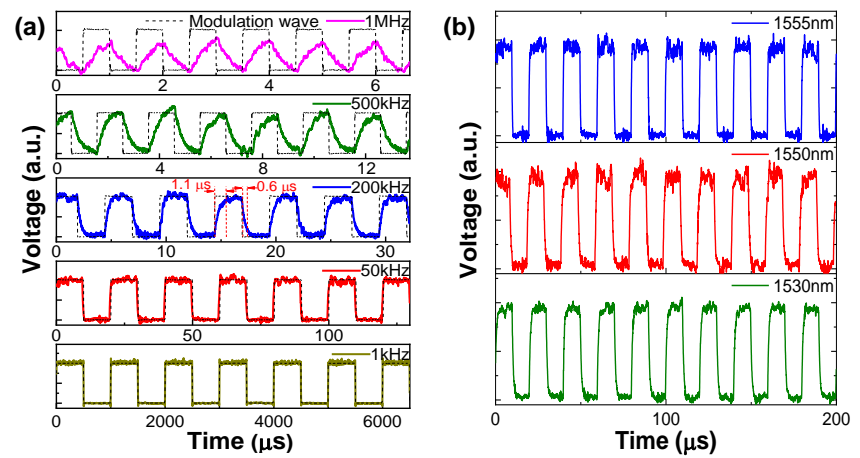

Fig. 4. Observations of multi-wavelength all-optical switching effects. (a) Temporal responses of the all-optical switching with modulation frequencies of $1 \mathrm{kHz}, 50 \mathrm{kHz}, 200 \mathrm{kHz}, 500 \mathrm{kHz}$, and $1 \mathrm{MHz}$. (b) Temporal response at 50 $\mathrm{kHz}$ modulation frequency with FBG filters at different wavelengths of around $1530 \mathrm{~nm}, 1550 \mathrm{~nm}$, and $1555 \mathrm{~nm}$.

We also evaluated the intensity of the output signal as a function of the pump power with a modulation frequency of 1 kHz, as shown in Fig. 5(a). It can be seen that the peak-to-peak value of the output waveform increases with the pump power, and demonstrates a linear relationship in the range of 0-30.7 $\mathrm{mW}$, as shown in Fig. 5(b). The results disclose that alloptically controlled spectrum linearly shifts with the lower pump power, and the FBG can be approximately considered as a linear filter. Owing to the pump wavelength equal to the dip wavelength of the cladding mode resonance, there is more light intensity leaked in the graphene layer to induce a strong absorption, resulting in a highly efficient pump even with only milliwatts pump power. It is noted that the decrease of the pump power will lead to a weak output signal caused by a low extinction ratio.

Our proposed graphene-integrated TFBG in a thin fiber is comparable to the modulation depth from other fiber-based alloptical switching devices by using the photothermal effect, and the modulation depth is mainly determined by the coupling intensity of the cladding mode resonances. However, the response time is much shorter, reduced to around $1 \mu \mathrm{s}$, as presented in Fig. 4. Compared with other schemes, including graphene/ $\mathrm{WS}_{2} /$ gold nanorods-coated microfibers [10-12], sidepolished twin-core fiber [13] or etched-FBG [15], and graphene-coated MZIs by external pumping [17, 29], the response rate of this device is enhanced by $\sim 3$ orders of magnitude. It could be attributed to the resonant-pump at a dip wavelength of the TFBG's cladding mode and the employment of the thin fiber, which will significantly enhance the accessible evanescent field and the pump efficiency. As a result, the cladding mode is easy to be modulated by interacting with the graphene and then fast thermo-optically induced refractive index change. The intrinsic thermal conductivity of graphene enables a very fast switching rate based on the thermal-optical effect $[22,23]$. In contrast with the non-resonant pump with a broadband light [16], by setting the pump wavelength at the cladding mode resonance, there is more light coupled into the graphene layer to heat the thin TFBG and endow it a highefficiency pump and fast heat-accumulation. Hence, the pump power can be reduced to a few milliwatts to achieve the switch of the intensity of the signal light, as shown in Fig. 5, which can also help to reduce the response time. It is therefore possible to shorten the response time to the order of microsecond, which is consistent with the reports by using much thinner fiber or onchip device in the experiment $[8,23]$. Moreover, compared with schemes using geometry-modified fibers embedded in interferometers, the integration of TFBG with a few-layer graphene offers a compact, robust structure near zero insertion loss, making them easier to be incorporated into a standard fiber-optic system for all-fiber signal processing.
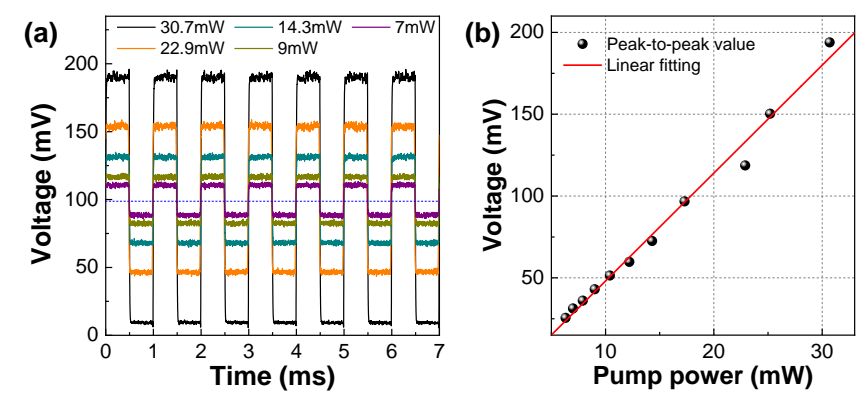

Fig. 5. (a) Temporal responses of the optical switching at $1 \mathrm{kHz}$ modulation frequency with pump powers of $7 \mathrm{~mW}, 9 \mathrm{~mW}, 14.3 \mathrm{~mW}, 22.9 \mathrm{~mW}$, and 30.7 $\mathrm{mW}$. (b) Peak-to-peak value versus the pump power.

\section{CONCLUSION}

In summary, we have demonstrated an all-optical switch in an all-fiber structure by integrating few-layer graphene onto a thin TFBG. It operates the thermo-optically induced refractive index change and then allows the spectral modulation of the cladding mode resonances in the graphene-integrated thin TFBG. The experimental results show that the response time of the device is reduced to around $1 \mu$ s, and the multi-wavelength operation is achieved in the range of tens of nanometers. Enlarging the spectral coverage and contrast would further extend the operation range and increase the modulation depth and rate by using a TFBG with much thinner fiber and larger tilted angle. The compact and robust structure is also much easier to integrate with standard fiber-optic systems. Additionally, this all-optical switching scheme can be applied to a photonic modulator or switch by integrating other twodimensional materials and/or fiber microstructures, which may 
bring many practical applications of graphene-like materials for signal processing.

\section{ACKNOWLEDGMENT}

The authors would like to thank the Analytical \& Testing Center of NPU for their assistance with the material and device characterizations, and Dr. Chao Wang (University of Kent) for helpful discussion.

\section{REFERENCES}

[1] G. T. Reed, G. Mashanovich, F. Y. Gardes, and D. J. Thomson, "Silicon optical modulators," Nat. Photon., vol. 4, no. 8, pp. 518-526, 2010.

[2] W. Li, B. Chen, C. Meng, W. Fang, Y. Xiao, X. Li, Z. Hu, Y. Xu, L. Tong, H. Wang, W. Liu, J. Bao, and Y. R. Shen, "Ultrafast all-optical graphene modulator," Nano Lett., vol. 14, no. 2, pp. 955-959, 2014.

[3] J.-H. Chen, B.-C. Zheng, G.-H. Shao, S.-J. Ge, F. Xu, and Y.-Q. Lu, "An all-optical modulator based on a stereo graphene-microfiber structure," Light Sci. Appl., vol. 4, pp. e360, 2015.

[4] M. Ono, M. Hata, M. Tsunekawa, K. Nozaki, H. Sumikura, H. Chiba, and M. Notomi, "Ultrafast and energy-efficient all-optical switching with graphene-loaded deep-subwavelength plasmonic waveguides," Nat. Photon., vol. 14, no. 1, pp. 37-43, 2020.

[5] J. Parra-Cetina, A. Kumpera, M. Karlsson, and P. A. Andrekson, "Phasesensitive fiber-based parametric all-optical switch," Opt. Express, vol. 23, no. 26, pp. 33426-33436, 2015

[6] Y. H. Kim, N. S. Kim, Y. Chung, U.-C. Paek, and W.-T. Han, "All-optical switching application based on optical nonlinearity of $\mathrm{Yb} 3+$ doped aluminosilicate glass fiber with a long-period fiber gratings pair," Opt. Express, vol. 12, no. 4, pp. 651-656, 2004.

[7] N. Nishizawa, and T. Goto, "Ultrafast all optical switching by use of pulse trapping across zero-dispersion wavelength," Opt. Express, vol. 11, no. 4, pp. 359-365, 2003.

[8] S. Yu, X. Wu, K. Chen, B. Chen, X. Guo, D. Dai, L. Tong, W. Liu, and Y. Ron Shen, "All-optical graphene modulator based on optical Kerr phase shift," Optica, vol. 3, no. 5, pp. 541-544, 2016.

[9] Z.-B. Liu, M. Feng, W.-S. Jiang, W. Xin, P. Wang, Q.-W. Sheng, Y.-G. Liu, D. N. Wang, W.-Y. Zhou, and J.-G. Tian, "Broadband all-optical modulation using a graphene-covered-microfiber," Laser Phys. Lett., vol. 10, no. 6, pp. 065901, 2013.

[10] X. Gan, C. Zhao, Y. Wang, D. Mao, L. Fang, L. Han, and J. Zhao, "Graphene-assisted all-fiber phase shifter and switching," Optica, vol. 2, no. 5, pp. 468-471, 2015.

[11] X. Yang, Q. Long, Z. Liu, Y. Zhang, J. Yang, D. Kong, L. Yuan, and K. Oh, "Microfiber interferometer integrated with Au nanorods for an allfiber phase shifter and switch," Opt. Lett., vol. 44, no. 5, pp. 1092-1095, 2019.

[12] K. Wu, C. Guo, H. Wang, X. Zhang, J. Wang, and J. Chen, "All-optical phase shifter and switch near $1550 \mathrm{~nm}$ using tungsten disulfide $\left(\mathrm{WS}_{2}\right)$ deposited tapered fiber," Opt. Express, vol. 25, no. 15, pp. 17639-17649, 2017.

[13] R. Chu, C. Guan, Y. Bo, J. Liu, J. Shi, J. Yang, P. Ye, P. Li, J. Yang, and L. Yuan, "Graphene decorated twin-core fiber Michelson interferometer for all-optical phase shifter and switch," Opt. Lett., vol. 45, no. 1, pp. 177$180,2020$.

[14] C. Gao, L. Gao, T. Zhu, and G. Yin, "Incoherent optical modulation of graphene based on an in-line fiber Mach-Zehnder interferometer," Opt. Lett., vol. 42, no. 9, pp. 1708-1711, 2017.

[15] X. Gan, Y. Wang, F. Zhang, C. Zhao, B. Jiang, L. Fang, D. Li, H. Wu, Z. Ren, and J. Zhao, "Graphene-controlled fiber Bragg grating and enabled optical bistability," Opt. Lett., vol. 41, no. 3, pp. 603-606, 2016.

[16] F. Liu, M. Qi, T. Guo, and J. Albert, "Saturable absorption and bistable switching of single mode fiber core-guided light by a $6 \mathrm{~nm}$-thick, few layers graphene coating on the cladding surface," Ann. Phys., vol. 532, no. 6, pp. 2000157, 2020.

[17] T. Hao, Z. Chang, and K. S. Chiang, "Externally pumped low-loss graphene-based fiber Mach-Zehnder all-optical switches with $\mathrm{mW}$ switching powers," Opt. Express, vol. 27, no. 4, pp. 4216-4225, 2019.

[18] B. Jiang, Z. Hao, D. Feng, K. Zhou, L. Zhang, and J. Zhao, "Hybrid grating in reduced-diameter fiber for temperature-calibrated highsensitivity refractive index sensing," Appl. Sci., vol. 9, no. 9, pp. 1923, 2019.
[19] C. Caucheteur, T. Guo, F. Liu, B.-O. Guan, and J. Albert, "Ultrasensitive plasmonic sensing in air using optical fibre spectral combs," Nat. Commun., vol. 7, pp. 13371, 2016.

[20] F. Liu, X. Zhang, T. Guo, and J. Albert, "Optical detection of the percolation threshold of nanoscale silver coatings with optical fiber gratings," APL Photon., vol. 5, no. 7, pp. 076101, 2020.

[21] S. Cai, F. Liu, R. Wang, Y. Xiao, K. Li, C. Caucheteur, and T. Guo, "Narrow bandwidth fiber-optic spectral combs for renewable hydrogen detection," Sci. China Inf. Sci., vol. 63, no. 12, pp. 222401, 2020.

[22] S. Gan, C. Cheng, Y. Zhan, B. Huang, X. Gan, S. Li, S. Lin, X. Li, J. Zhao, H. Chen, and Q. Bao, "A highly efficient thermo-optic microring modulator assisted by graphene," Nanoscale, vol. 7, no. 47, pp. 2024920255, 2015.

[23] Z. Yu, Y. Wang, B. Sun, Y. Tong, J.-B. Xu, H. K. Tsang, and X. Sun, "Hybrid 2D-material photonics with bound states in the continuum," $A d v$. Opt. Mater., vol. 7, no. 24, pp. 1901306, 2019.

[24] M. Qi, Y. Zhou, F. Hu, X. Xu, W. Li, A. Li, J. Bai, and Z. Ren, "Improving Terahertz sheet conductivity of graphene films synthesized by atmospheric pressure chemical vapor deposition with acetylene," J. Phys. Chem. C, vol. 118, no. 27, pp. 15054-15060, 2014.

[25] B. Jiang, G. Yin, K. Zhou, C. Wang, X. Gan, J. Zhao, and L. Zhang, "Graphene-induced unique polarization tuning properties of excessively tilted fiber grating," Opt. Lett., vol. 41, no. 23, pp. 5450-5453, 2016.

[26] R. R. Nair, P. Blake, A. N. Grigorenko, K. S. Novoselov, T. J. Booth, T. Stauber, N. M. R. Peres, and A. K. Geim, "Fine structure constant defines visual transparency of graphene," Science, vol. 320, no. 5881, pp. 1308$1308,2008$.

[27] Y. Wang, B. Jiang, S. Das, Q. Zhao, X. Gan, and J. Zhao, "All-optically controlled slow and fast lights in graphene-coated tilted fiber Bragg grating," Appl. Phys. Express, vol. 12, no. 7, pp. 072010, 2019.

[28] X. Wang, W. Jin, Z. Chang, and K. S. Chiang, "Buried graphene electrode heater for a polymer waveguide thermo-optic device," Opt. Lett., vol. 44, no. 6, pp. 1480-1483, 2019.

[29] Y. K. Ma, J. C. Tan, Q. Q. Gu, X. X. Chen, H. Zhang, H. M. Yang, G. L. Deng, H. Zhou, and S. H. Zhou, "Multilayer graphene improved interface thermal property for all-optical controlled fiber interferometer," Laser Phys. Lett., vol. 17, no. 6, pp. 065104, 2020. 